\title{
Processing a Biological Tissue from Cryo-SEM to Replica
}

\author{
Nadezda Vaskovicova $^{1}$, Kamila Hrubanova ${ }^{1}$ and Vladislav Krzyzanek ${ }^{1}$ \\ ${ }^{1 .}$ Institute of Scientific Instruments of the CAS, v.v.i., Brno, Czech Republic.
}

Cryo-SEM is a technique suitable for observation of frozen biological samples. The cryo-preparation of tissue fragments without using cryo-protectants can destroy intracellular compartments and cytoplasm. On the other hand the use of cryoprotectants can prevent deeper etching of the medium and thus it often leads to more complicated detection of cell membranes, which are important for orientation in the sample during its observation. This can be managed by shadowing of the fractured structures by platinum and it may help to heighten the structures as is done in production of replicas which can give more detail at higher magnification. Here we report how the replicas can be prepared in the device ACE600 (Leica microsystems) equipped with the cryo-attachement and two guns for metal sputtering and carbon evaporation, and the combination of cryo-SEM and replicas.

The pieces of the tissue (mouse stomach epithelium) were fixed by $3 \%$ glutaraldehyde, washed in PBS buffer and saturated by $20 \%$ glycerol in the fridge overnight. The pieces were placed on the freezefracture carriers and frozen in melting nitrogen. After transferring the frozen samples to ACE600, the tissues were fractured and etched for $13 \mathrm{~min}$ at $-100^{\circ} \mathrm{C}$. For investigation in the cryo-SEM the samples were sputtered by Pt of $2.0 \mathrm{~nm}$ followed later by additional $2.6 \mathrm{~nm}$ at the angle of $50^{\circ}$ (argon atmosphere of $8.1 \times 10^{-3} \mathrm{mbar}$ ). The frozen samples were transferred to the SEM Magellan 400 (FEI) by the shuttle VCT100 (Leica microsystem), and imaged with $1 \mathrm{keV}$ electrons (SE mode, low-dose) at the temperature of $-120^{\circ} \mathrm{C}$. For replicas, the samples were sputtered by $2.1 \mathrm{~nm} \mathrm{Pt}$, at an angle of $50^{\circ}$ and a pressure of $8.1 \times 10^{-3}$ mbar, followed by evaporation of $21 \mathrm{~nm} \mathrm{C}$ at an angle of $90^{\circ}$. After that the samples were melted at room temperature and the tissue remnants were separated from the carrier on the surface of the acid. The $50 \%$ sulfuric acid and $2 \%$ sodium hypochlorite were used for removing the biological remnants from the metal replicas. The replicas were viewed in the TEM Morgagni 268D (FEI).

The cryo-SEM images of the sample which was covered by $2 \mathrm{~nm}$ Pt (Figure 1A, B) show a better resolution among the individual cells and the extracellular space in low magnification in contrast with the sample without coating. But in detail of the cell organelles there can be seen the same destruction of the tissue due to electron beam damage (Figure 1B black arrows). The added layer of Pt to the final deposit of $4.6 \mathrm{~nm}$ allowed detecting more details in the fractured tissue and the details on individual cell organelles, distribution of intramembrane particles or cytoplasmic particles (Figure 1C, D). The replicas prepared in the ACE600 (Figure 1E, F) have good quality and provide detailed description of intracellular space or membrane-particles distribution. Unfortunately the replicas are very fragile. A very common effect is the ripping and twisting of the replicas when removing the biological remnants. It means that the images from cryo-SEM can be only evidence of the examined biological structure.

The combination of both techniques, cryo-SEM and replica, can serve for documentation of large biological objects with details at the surface of membrane ultrastructure, and can be prepared by a modern cryo-preparation unit equipped just with metal sputtering equipment such as ACE600. [1]

References:

[1] The research was supported by MEYS CR (LO1212) and its infrastructure by MEYS CR and EC (CZ.1.05/2.1.00/01.0017). 

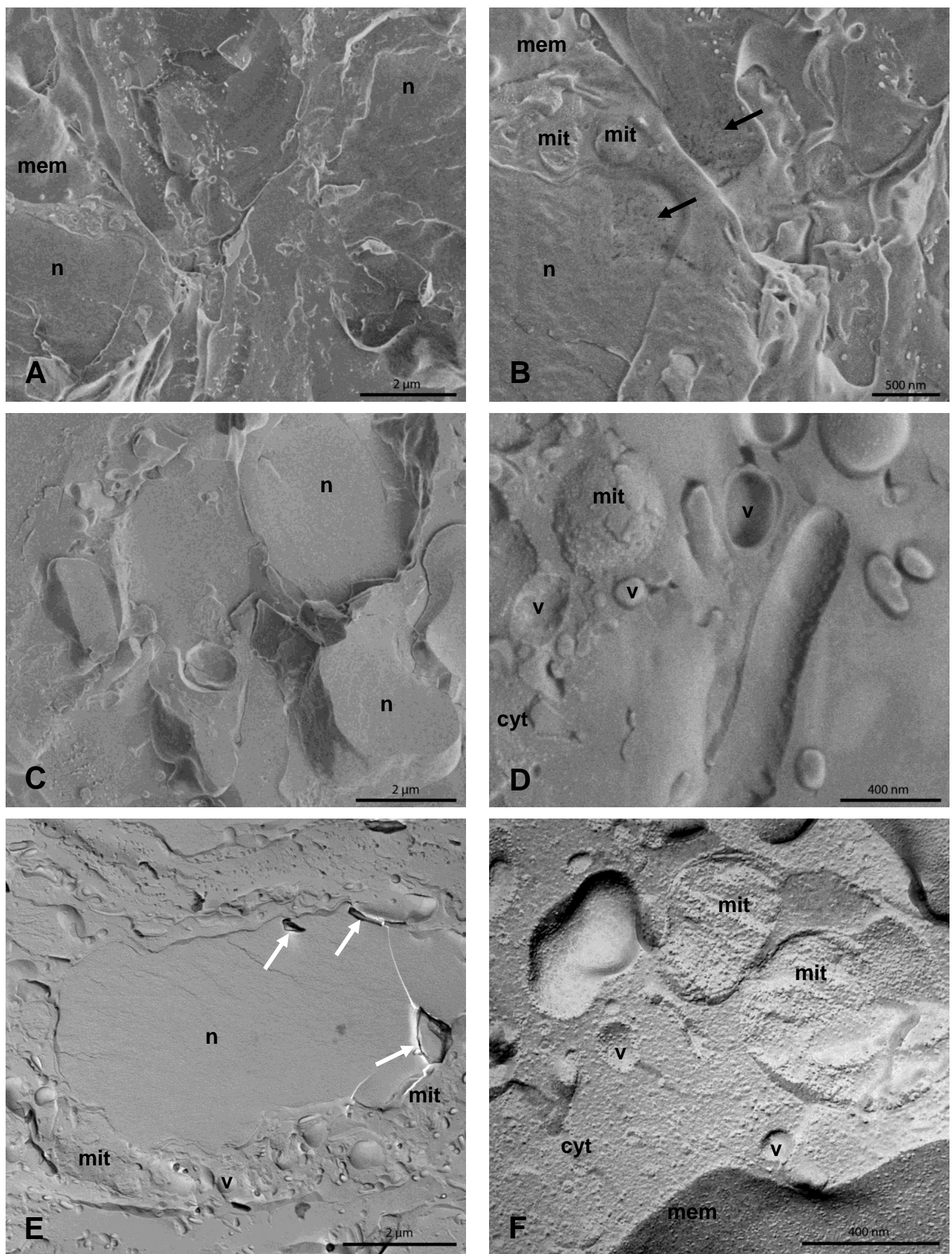

Figure 1. A, B Cryo-SEM, $2 \mathrm{~nm}$ Pt, black arrows destruction of the sample by electron beam; C, D cryo-SEM, $4.6 \mathrm{~nm} \mathrm{Pt;} \mathrm{E,} \mathrm{F} \mathrm{replicas,} 2.1 \mathrm{~nm} \mathrm{Pt,} 21 \mathrm{~nm} \mathrm{C}$, white arrow pieces of destructed replica; $\mathbf{n}$ nucleus, mit mitochondrion, mem plasma membrane, $\mathbf{v}$ vesicle, cyt particles in cytoplasm. 